\title{
TRATAMENTO DO EFLUENTE DO BIODIESEL UTILIZANDO A ELETROCOAGULAÇÃO/FLOTAÇÃO: INVESTIGAÇÃO DOS PARÂMETROS OPERACIONAIS
}

Janaina Moreira de Meneses*, Robelsa de Fátima Vasconcelos, Thalys de Freitas Fernandes e Gilmar Trindade de Araújo Unidade Acadêmica de Engenharia Química, Universidade Federal de Campina Grande, Rua Aprígio Veloso, 882, Bloco CM, 58429-140 Campina Grande - PB, Brasil

Recebido em 15/9/10; aceito em 28/7/11; publicado na web em 26/9/11

TREATMENT OF BIODIESEL WASTEWATER BY ELECTROCOAGULATION/FLOTATION PROCESS: INVESTIGATION OF OPERATIONAL PARAMETERS. Electrocoagulation/flotation process was applied to treat biodiesel wastewater using aluminium electrodes. Firstly, a literature survey was conducted to choose the process variables and then, operational parameters including initial $\mathrm{pH}$, electrode distance and reaction time were tested. Experimental results showed the best parameter that can be used in a factorial design for further studies. The results indicate that electrocoagulation/flotation is very efficient to reduce oil and grease, the effluent was very clear after treatment and small amount of sludge was produced.

Keywords: electrocoagulation; biodiesel wastewater; operational parameters.

\section{INTRODUÇÃO}

A dependência do petróleo e a poluição gerada pelo óleo diesel são as grandes desvantagens do uso deste combustível, o que vem estimulando a busca por fontes energéticas alternativas. ${ }^{1} \mathrm{O}$ biodiesel é um combustível alternativo ao óleo diesel, é produzido pela reação química entre um óleo vegetal ou gordura animal com um álcool. ${ }^{2} \mathrm{O}$ Brasil está entre os maiores produtores e consumidores de biodiesel do mundo, com uma produção anual, em 2010, de 2,4 bilhões de litros e uma capacidade instalada, no mesmo ano, para cerca de 5,8 bilhões de litros. ${ }^{3}$

Diversos métodos de produção de biodiesel vêm sendo desenvolvidos. ${ }^{4} \mathrm{O}$ método de obtenção do biodiesel que o governo brasileiro incentiva é o de transesterificação, que consiste na reação química de triglicerídeos com alcoóis (metanol ou etanol) na presença de um catalisador (ácido, básico ou enzimático), resultando na substituição do grupo éster do glicerol pelo grupo etanol ou metanol. ${ }^{1}$ Em escala industrial, catalisador alcalino é o mais utilizado devido a suas condições operacionais adequadas: baixo custo, fácil instalação e, acima de tudo, sua alta taxa de reação proporciona uma diminuição no tamanho de equipamento e capital. Além disto, o metanol é o álcool mais comumente utilizado devido seu baixo custo e suas vantagens físico-químicas (compostos polares e álcool de cadeia curta). ${ }^{5} \mathrm{No}$ Brasil, das 48 empresas que produziram biodiesel (B100) em 2009, 42 adotaram a rota metílica (uso de metanol), representando $94,7 \%$ desta produção. $\mathrm{O}$ óleo de soja foi a principal matéria-prima para a produção de biodiesel (B100), a segunda matéria-prima no ranking de produção das usinas foi o sebo bovino, seguido pelo óleo de algodão. ${ }^{6}$

Após a reação de transesterificação, o glicerol é removido dos ésteres metílicos por decantação, no entanto, para atender os critérios de qualidade do biodiesel é necessária a etapa de purificação, para a remoção de qualquer quantidade de resíduo que tenha ficado no biodiesel, e para isto é realizada uma lavagem aquosa. ${ }^{7}$ Nesta etapa, a água remove qualquer catalisador remanescente, sabões, sais, metanol e glicerina livre do biodiesel. ${ }^{2}$ A demanda atual de biodiesel é atendida pelo processo convencional de transesterificação com catalisador alcalino. Portanto, diante da crescente expansão da produção de biodiesel em plantas industriais que utilizam o processo

*e-mail: janameneses@hotmail.com convencional, verifica-se a necessidade de tratar o efluente gerado durante este processo produtivo, ${ }^{8}$ de forma que adquira características dentro dos padrões de lançamento de efluentes presentes na Resolução CONAMA n ${ }^{\circ} 357$ de 17/6/2005. ${ }^{9}$ Segundo Suehara et al.,${ }^{5}$ utilizando o processo de transesterificação, para cada $100 \mathrm{~L}$ de biodiesel produzido, são gerados $20 \mathrm{~L}$ de efluente; conforme Kolesárová et al., ${ }^{10}$ dependendo do método de lavagem aplicado até $300 \mathrm{~L}$ de efluente podem ser gerados para cada $100 \mathrm{~L}$ de biodiesel produzidos. De Boni et al. ${ }^{11}$ verificaram que são necessários no mínimo $3 \mathrm{~L}$ de água para cada litro de biodiesel produzido, Granjeiro ${ }^{12}$ também utilizou $3 \mathrm{~L}$ de água deionizada para lavagem de $1 \mathrm{~L}$ de biodiesel. Este efluente gerado possui um alto valor de $\mathrm{pH}$, devido aos níveis significativos residuais de catalisador alcalino, e contem um alto teor de óleo e de sólidos e baixa concentração de nitrogênio. Juntos, estes componentes inibem o crescimento da maioria dos micro-organismos, tornando este efluente difícil de degradar naturalmente. ${ }^{8}$

Como forma de tratamento do efluente do biodiesel, pesquisadores vêm utilizando diversas técnicas. Suehara et al. ${ }^{4}$ realizaram um tratamento biológico e verificaram que este tipo de tratamento é difícil devido à composição do efluente, que não é apropriado ao crescimento microbiano. Para aplicar este tratamento, os autores modificaram o $\mathrm{pH}$ e adicionaram diversos nutrientes ao efluente para facilitar a degradação do óleo. ${ }^{4}$ Jaruwat et al. ${ }^{8}$ conduziram um tratamento do efluente do biodiesel em dois estágios; no primeiro, o biodiesel foi quimicamente recuperado do efluente utilizando-se ácido sulfúrico como doador de prótons e subsequente separação natural das fases. No segundo, a fase aquosa resultante foi sujeita ao processo de eletro-oxidação. O processo combinado removeu completamente DQO, óleos e graxas do efluente, e reduziu o nível de DBO em 95\%.

O tratamento eletroquímico vem sendo utilizado na descontaminação de diversos efluentes e testado com sucesso para tratar esgotos sanitários, efluentes de restaurantes, remover óleos e graxas de efluente industrial, remoção de óleo da emulsão óleo/água, remoção de corantes, entre outros. ${ }^{13-18} \mathrm{Um}$ dos processos eletroquímicos que vem se destacando no meio cientifico é o processo de eletrocoagulação/flotação, onde um reator eletroquímico é o centro das reações de coagulação.

O processo de eletrocoagulação tem atraído uma grande atenção no tratamento de efluentes industriais devido a sua versatilidade e compatibilidade ambiental. Esta técnica tem algumas vantagens 
quando comparada aos métodos tradicionais, tais como simplicidade de equipamento, facilidade de operação, menor tempo de retenção, ausência de adição de produtos químicos, rápida sedimentação dos flocos eletrogerados e menor produção de lodo. ${ }^{19}$

No processo de eletrocoagulação, íons são produzidos in situ e isto envolve três estágios sucessivos: formação do coagulante pela oxidação eletrolítica do 'eletrodo de sacrifício', desestabilização dos contaminantes, suspensão das partículas e quebra de emulsões e, agregação das fases desestabilizadas para formar flocos. A água também é eletrolisada em uma reação paralela, produzindo pequenas bolhas de oxigênio no ânodo e hidrogênio no catodo. Essas bolhas atraem as partículas floculadas e, por meio de flutuação natural, os poluentes floculados flotam até a superfície. ${ }^{20}$

A eletrofloculação é a combinação dos processos de eletrocoagulação e eletroflotação, já que este processo consiste na geração de íons metálicos para a posterior formação do agente coagulante e a eletroflotação, na geração dos gases hidrogênio e oxigênio pela eletrólise da água. ${ }^{18}$

As reações que ocorrem durante a eletrocoagulação, utilizando eletrodos de alumínio são: ${ }^{15}$

$$
\begin{gathered}
\mathrm{Al} \rightarrow \mathrm{Al}^{3+}+3 e^{-}(\text {ânodo }) \\
2 \mathrm{H}_{2} \mathrm{O}+2 e^{-} \rightarrow \mathrm{H}_{2}+2 \mathrm{OH}^{-}(\text {cátodo })
\end{gathered}
$$

Segundo alguns autores, ${ }^{14,20,21}$ quando o potencial anódico é suficientemente alto, reações secundárias podem ocorrer, especialmente a evolução do oxigênio (Equação 3):

$$
2 \mathrm{H}_{2} \mathrm{O} \rightarrow \mathrm{O}_{2}+4 \mathrm{H}^{+}+4 e^{-}
$$

Os íons alumínio $\left(\mathrm{Al}^{3+}\right)$ produzidos pela dissolução eletrolítica do anodo (Equação 1) sofrem imediatamente reações de hidrólise espontânea, o que gera várias espécies monoméricas de acordo com a seguinte sequência: ${ }^{19}$

$$
\begin{gathered}
\mathrm{Al}^{3+}+\mathrm{H}_{2} \mathrm{O} \rightarrow \mathrm{Al}(\mathrm{OH})^{2+} \\
\mathrm{Al}(\mathrm{OH})^{2+}+\mathrm{H}_{2} \mathrm{O} \rightarrow \mathrm{Al}(\mathrm{OH})_{2}^{+}+\mathrm{H}^{+} \\
\mathrm{Al}(\mathrm{OH})_{2}^{+}+\mathrm{H}_{2} \mathrm{O} \rightarrow \mathrm{Al}(\mathrm{OH})_{3}+\mathrm{H}^{+}
\end{gathered}
$$

Este trabalho teve como objetivo realizar um estudo inicial sobre o processo de eletrocoagulação, verificando quais as principais variáveis que influenciam no processo e avaliar alguns parâmetros operacionais, como pH, tempo de tratamento e distância entre eletrodos, para sua fixação em trabalhos futuros sobre eletrocoagulação em efluente do biodiesel.

\section{PARTE EXPERIMENTAL}

Segundo Barros Neto et al., ${ }^{22}$ a essência de um bom planejamento consiste em projetar um experimento de forma que ele seja capaz de fornecer exatamente o tipo de informação que se esteja procurando. Portanto, realizou-se inicialmente uma pesquisa bibliográfica de modo a se fazer uma triagem dos fatores que influenciam no processo de eletrocoagulação e descartar as variáveis não significativas, evitando assim um maior número de ensaios no laboratório e tornando os experimentos menos onerosos.

Uma célula eletrolítica pode ser projetada de diversas formas, ${ }^{23}$ além disto, vários fatores podem influenciar as reações de eletrólise e, consequentemente, o resultado obtido no tratamento de um resíduo líquido. Dentre os fatores que devem ser avaliados na especificação de um projeto estão: tipo de resíduo, temperatura de reação, tempo de tratamento, faixa de $\mathrm{pH}$, condutividade do eletrólito, distância entre os eletrodos, tensão elétrica, corrente elétrica e material dos eletrodos. ${ }^{24}$

Diversos trabalhos, publicados na última década, relacionam as variáveis que influenciam o processo de eletrocoagulação e que podem ser tomados como uma base inicial à pesquisa e desenvolvimento deste processo. ${ }^{14,25-27}$

Em um estudo experimental o ideal é controlar o maior número possível dessas variáveis, no entanto, como isso não é sempre possível, devem-se escolher aquelas que mais influenciam no desempenho da célula eletroquímica e, consequentemente, no processo de eletrocoagulação. Desta forma, fixou-se para este trabalho o material dos eletrodos (alumínio), as dimensões dos eletrodos (10 x 5 x $1 \mathrm{~mm}$ ), volume do efluente a ser tratado $(0,5 \mathrm{~L})$ e temperatura do efluente bruto (temperatura ambiente $-28^{\circ} \mathrm{C}$ ).

Como a eficiência de um sistema de eletrocoagulação/flotação é refletida na remoção de poluentes e potência elétrica e/ou consumo químico, ${ }^{28}$ os parâmetros espaçamento entre os eletrodos, $\mathrm{pH}$ do efluente e tempo de eletrólise foram testados e avaliados. Os parâmetros inicialmente fixados para a avaliação do processo de eletrocoagulação/flotação são mostrados na Tabela 1.

Tabela 1. Parâmetros testados nos experimentos

\begin{tabular}{ccccc}
\hline Ensaio & $\mathrm{pH}_{\mathrm{i}}$ & $\begin{array}{c}\text { Distânc. } \\
\text { eletrodos (mm) }\end{array}$ & $\begin{array}{c}\text { Tempo } \\
\text { eletrólise (min) }\end{array}$ & $\begin{array}{c}\text { Voltagem } \\
\text { aplicada (v) }\end{array}$ \\
\hline $\mathrm{A}$ & 5,33 & 8 & 40 & 4,8 \\
$\mathrm{~B}$ & 7,92 & 4 & 20 & 3,6 \\
$\mathrm{C}$ & 5,25 & 6 & 40 & 3,5 \\
$\mathrm{D}$ & 5,26 & 4 & 40 & 3,5 \\
$\mathrm{E}$ & 2,50 & 4 & 60 & 3,6 \\
\hline
\end{tabular}

\section{Material do eletrodo utilizado}

Dentre os diversos tipos de materiais que podem ser escolhidos como eletrodo, o alumínio é o mais indicado, por apresentar baixo custo e estar facilmente disponível no mercado. Além disto, conforme Ferreira, ${ }^{29}$ outra vantagem deste material é que os eletrodos de alumínio não deixam cor no efluente resultante do tratamento e é bastante eficaz, devido a sua capacidade superior de adsorção do hidróxido de alumínio em relação aos hidróxidos de ferro (II) e (III) quando se usam eletrodos de ferro.

\section{Aparato experimental}

Os experimentos de eletrocoagulação/flotação foram conduzidos em um reator monopolar em batelada. No reator de vidro com capacidade de 0,6 L, um par de eletrodos de alumínio de área de 50 $\mathrm{cm}^{2}$ foi inserido verticalmente e ligado a uma fonte alimentadora de corrente contínua. As placas de alumínio foram separadas por um parafuso e espaçadores de plástico. Na Figura 1 é exibido o sistema experimental utilizado.

Inicialmente os eletrodos foram inseridos no reator e conectados à fonte de alimentação, em seguida o efluente foi adicionado ao reator e o sistema foi mantido sob agitação constante por meio de agitador magnético; $\mathrm{NaCl}$ foi adicionado ao efluente como forma de melhorar a condutividade do eletrólito, alcançando um valor médio de $2,5 \mathrm{mS} / \mathrm{cm}$ e, por fim, foi aplicada uma voltagem ao sistema. Depois de transcorrido o tempo de tratamento pré-determinado, o lodo gerado foi removido e o efluente tratado foi analisado. Antes e após cada ensaio, os eletrodos foram lavados, secados em estufa e depois pesados. 




Figura 1. Sistema de eletrocoagulação/flotação: 1) fonte de alimentação; 2) agitador magnético; 3) barra magnética; 4) eletrodos; 5) reator eletrolítico

\section{Análises dos efluentes tratados e do efluente bruto}

As análises físico-químicas realizadas nos efluentes tratado e bruto foram $\mathrm{pH}$, condutividade, sólidos totais, óleos e graxas.

Para medir o pH realizou-se medição instrumental utilizando o pH-metro digital microprocessado, marca Micronal, com faixa de operação de $-2,000$ a 16,00. A condutividade elétrica foi mensurada pelo condutivímetro, marca Digimed, com faixa de medição de 0,01 $\mathrm{S} / \mathrm{cm}$ a $200 \mathrm{mS} / \mathrm{cm}$. As análises de sólidos totais foram realizadas através do método gravimétrico de determinação de sólidos totais seco, de 103 a $105{ }^{\circ} \mathrm{C}$, descrito no Standard Methods for the Examination of Water \& Wastewater. ${ }^{30}$ Para a determinação de óleos e graxas utilizou-se o método gravimétrico de extração por n-hexano para análise em águas, conforme descrito no Standard Methods for the Examination of Water \& Wastewater ${ }^{30}$ Nesta na determinação de óleos e graxas não se mede uma quantidade absoluta de uma substância específica, mas se determina quantitativamente a substância solúvel em um solvente. Isto quer dizer que são consideradas graxas os ácidos graxos, as gorduras animais, os sabões, as graxas, as ceras, os óleos vegetais e qualquer outro tipo de substância que o solvente consegue extrair de uma amostra acidificada, que não se volatilizam durante a evaporação. ${ }^{31}$

\section{Características do efluente}

O efluente foi gerado durante a etapa de purificação do biodiesel, produzido pelo processo de transesterificação alcalina do óleo de algodão comercial em escala laboratorial, realizado no Laboratório de Química de Biomassa, da Unidade Acadêmica de Engenharia Química, na Universidade Federal de Campina Grande.

Após a remoção da glicerina do produto formado (biodiesel), água aquecida a $50{ }^{\circ} \mathrm{C}$ foi inserida no balão de separação para remover as impurezas que ficaram no biodiesel após a reação de transesterificação com metanol. Desta forma, após a lavagem do biodiesel, verificou-se um efluente turvo, de cor branca, com uma lâmina de óleo na superfície do efluente. As análises mostram um efluente com $\mathrm{pH}$ elevado, igual a 10, alto teor de óleos e graxas $(9.469 \mathrm{mg} / \mathrm{L})$ e de sólidos totais $(6.500 \mathrm{mg} / \mathrm{L})$, bem como baixo valor de condutividade $(0,65 \mathrm{mS} / \mathrm{cm})$.

\section{Variação do pH do efluente bruto}

Realizaram-se 5 experimentos para avaliar o efeito do $\mathrm{pH}$ no processo de eletrocoagulação. Para realizar a modificação no $\mathrm{pH}$ utilizou-se de solução de $\mathrm{NaOH}(1 \mathrm{~N})$ e $\mathrm{HCl}(1 \mathrm{~N})$. Os ensaios A, C e D tiveram um pH inicial de 5 e mesmo tempo de tratamento de 40 min, no entanto, foram usados diferentes espaçamentos dos eletrodos, para se verificar a influência deste parâmetro nos resultados. Já os ensaios B e E tiveram mesmo espaçamento dos eletrodos (4 mm) e diferentes tempos de tratamento ( 20 e $60 \mathrm{~min}$, respectivamente) para se verificar o comportamento do processo no valor de $\mathrm{pH}$ determinado $\left(\mathrm{pH}_{\mathrm{B}}=7,92\right.$ e $\left.\mathrm{pH}_{\mathrm{E}}=2,50\right)$.

\section{Desgaste dos eletrodos}

Conforme a Lei de Faraday, o consumo de elétrons é associado à quantidade total de substâncias reagentes. Na eletrocoagulação, este fato está diretamente relacionado ao desgaste do eletrodo (corrosão) no processo de geração do agente coagulante. Isto significa que a geração de alumínio em solução está intimamente relacionada à carga, e esta pode ser controlada pela corrente obtida. Assim, a corrente medida por um multímetro no processo de eletrocoagulação é, a princípio, proporcional à quantidade de alumínio carregado gerado em solução. A massa do eletrodo consumida $\left(\mathrm{m}_{\mathrm{el}}\right)$ ou massa teórica $\left(\mathrm{m}_{\mathrm{teo}}\right)$, em g, durante a eletroflotação, pode ser calculada pela Equação $7 .^{32}$

$$
m_{t e o}=\frac{i \times t \times M}{F \times n}
$$

onde i é a corrente (A); t, o tempo de aplicação da corrente (s); M, a massa molar do elemento predominante do eletrodo $(\mathrm{g} / \mathrm{mol}) ; \mathrm{n}, \mathrm{o}$ número de elétrons envolvidos na reação de oxidação do elemento do anodo $\left(\mathrm{n}_{\mathrm{Al}}=3\right)$ e $\mathrm{F}$, a constante de Faraday $\left(9,65 \times 10^{4} \mathrm{C} / \mathrm{mol}\right)$.

\section{RESULTADOS E DISCUSSÃO}

A Figura 2 ilustra o aspecto visual dos efluentes tratado e bruto. Verifica-se que após o tratamento, os efluentes A e D apresentaram melhor aspecto visual, estando transparentes, já nos ensaios B, D e E verifica-se um visual um pouco turvo.

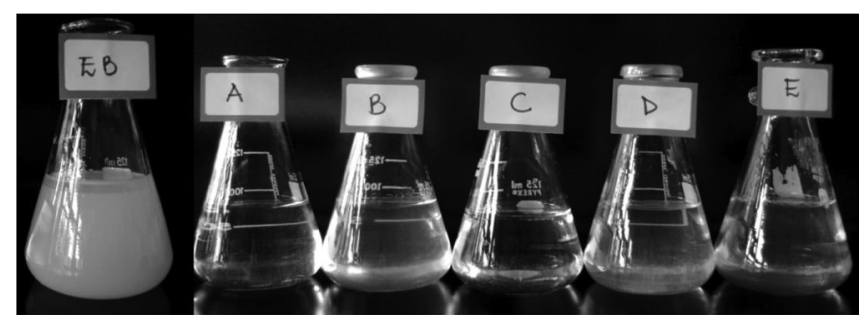

Figura 2. Fotografia em fundo escuro das amostras do efluente bruto (EB)e dos efluentes tratados referentes a cada ensaio realizado

\section{Variação do pH do efluente}

Diversos autores observaram que o $\mathrm{pH}$ afluente ao reator de eletroflotação é um importante fator operacional que influencia na performance do processo eletroquímico. ${ }^{14,32} \mathrm{Na}$ Figura 3 é observada a relação entre o $\mathrm{pH}$ do efluente antes e após o tratamento. Observouse que ocorre um aumento do $\mathrm{pH}$ do efluente durante o processo de eletrocoagulação/flotação. Vários autores atribuíram esse aumento do pH à evolução do hidrogênio no catodo, conforme a Equação 2.15,23,31 Mohouedhen et al..$^{33}$ estudaram o processo de eletrocoagulação utilizando eletrodos de alumínio e verificaram que quando o pH inicial é ácido o valor do $\mathrm{pH}$ final aumenta, já quando o $\mathrm{pH}$ inicial é alcalino, o $\mathrm{pH}$ final diminui. Os autores ainda verificaram neste processo de eletrocoagulação que em soluções altamente ácidas ( $\mathrm{pH}$ 2) ou alcalinas ( $\mathrm{pH}$ 12), o pH permanece inalterado.

De acordo com Chen et al., ${ }^{14} \mathrm{o}$ aumento de $\mathrm{pH}$ pode ter ocorrido a partir de outros mecanismos, como a transferência de $\mathrm{CO}_{2}$, pois o $\mathrm{CO}_{2}$ é super saturado em eletrólito aquoso ácido e pode ser liberado do meio devido à agitação causada pelas bolhas de $\mathrm{H}_{2}$, causando então o aumento do $\mathrm{pH}$. 


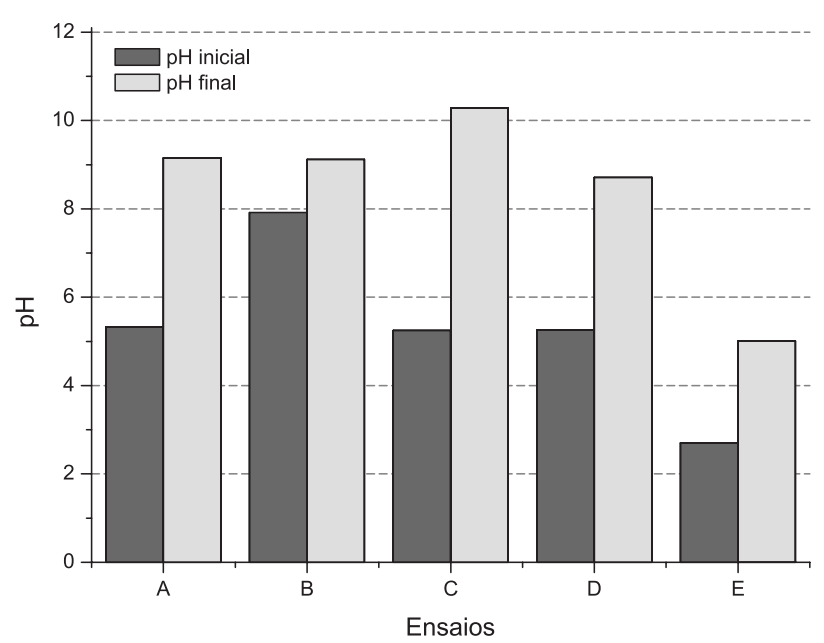

Figura 3. Variação do pH do efluente antes e após o processo de eletrocoagulação

\section{Lodo gerado}

No processo de eletrocoagulação, muitos dos poluentes são separados do efluente e flotados para a superfície na forma de lodo. O lodo produzido é proporcional às características do efluente bruto, sólidos sedimentáveis, matéria desestabilizada por coagulação, é proporcional à densidade de corrente e ao tempo de tratamento. ${ }^{19}$

Nos ensaios C e D foi observada, ao final do tratamento, uma espessa camada de espuma na superfície do reator; já nos ensaios A, $\mathrm{B}$ e E, a camada de espuma formada foi fina.

\section{Alumínio liberado durante o processo de eletrocoagulação/ flotação}

O estudo do alumínio liberado durante a eletrólise (para eletrodos de alumínio) como dosador de coagulante é um parâmetro chave no processo de eletrocoagulação. Na verdade, desde que a coagulação é alcançada pela neutralização de carga e desestabilização dos coloides carregados negativamente pela hidrólise catiônica dos produtos de $\mathrm{Al}^{3+}$, o excesso de coagulante pode dar uma carga reversa e reestabilização dos coloides. ${ }^{33}$

No processo eletrolítico ocorre a corrosão dos eletrodos, logo, de acordo com a Lei de Faraday, ocorre uma perda de massa dos eletrodos; o valor teórico desta massa consumida no processo pode ser verificada pela Equação 7. A quantidade de alumínio liberada dos eletrodos durante os ensaios experimentais foi calculada através da diferença dos pesos dos eletrodos antes e após cada ensaio; o resultado pode ser verificado na Figura 4. Observa-se em todos os ensaios que o valor experimental do alumínio liberado dos eletrodos foi maior que o valor teórico. Este aumento do valor da massa do alumínio consumida experimental $\left(\mathrm{M}_{\text {exp }}\right)$ quando comparado com o valor teórico $\left(\mathrm{M}_{\text {teo }}\right)$ pode ser atribuído ao fato do alumínio total consumido ser a soma do alumínio dissolvido eletricamente (Faraday) e quimicamente, pela dissolução do cátodo, devido ao ataque químico pelos íons hidroxila gerados durante a redução da água (Equação 2), especialmente em altos valores de $\mathrm{pH}$, como provado por diversos autores, ${ }^{14,19,34,35}$ e isto pode ser observado pela perda de peso do cátodo nos experimentos.

Os ensaios A e B foram os que obtiveram menor consumo de eletrodos; nestes dois ensaios observou-se que a corrente requerida foi menor quando comparada aos demais ensaios; além disto, no ensaio B o tempo de tratamento foi de $20 \mathrm{~min}$, o que também influencia no consumo de eletrodos. No ensaio E o tempo de tratamento foi de 60

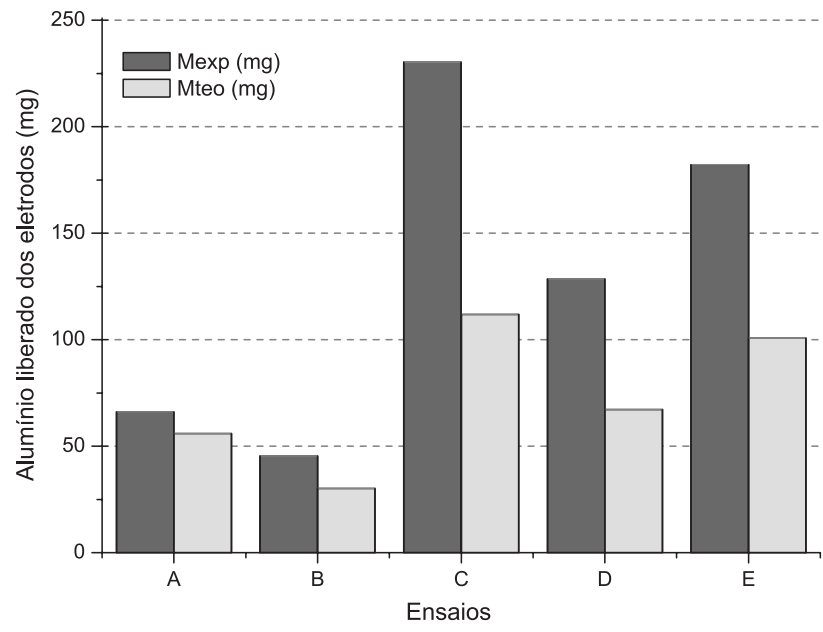

Figura 4. Alumínio liberado dos eletrodos durante o processo de eletrocoagulação/flotação

min e, consequentemente, uma quantidade maior de alumínio foi consumida. No ensaio $\mathrm{C}$ o elevado consumo pode ser atribuído ao elevado valor do $\mathrm{pH}$ final, pois um $\mathrm{pH}$ alcalino conduz a um consumo maior do cátodo devido ao ataque dos íons hidroxila (Equação 2); outro fator que influenciou no ensaio $\mathrm{C}$ foi a corrente requerida, que foi maior do que nos outros. Segundo Vik et al., ${ }^{36}$ um aumento na densidade de corrente produz uma maior quantidade de coagulante $\left(\mathrm{Al}^{3+}\right)$ liberado dos eletrodos. Simultaneamente, a taxa do gás hidrogênio no catodo também é aumentada.

\section{Sólidos totais}

Segundo a APHA, a determinação da concentração de sólidos totais envolve todos os compostos particulados e dissolvidos como metais, sais inorgânicos, óleos e graxas e matéria orgânica presentes no efluente. ${ }^{30}$ Essa determinação é importante, pois fornece informações sobre a quantidade de resíduos presentes nos efluentes. A Figura 5 apresenta a concentração de sólidos totais em função do tipo de ensaio realizado.

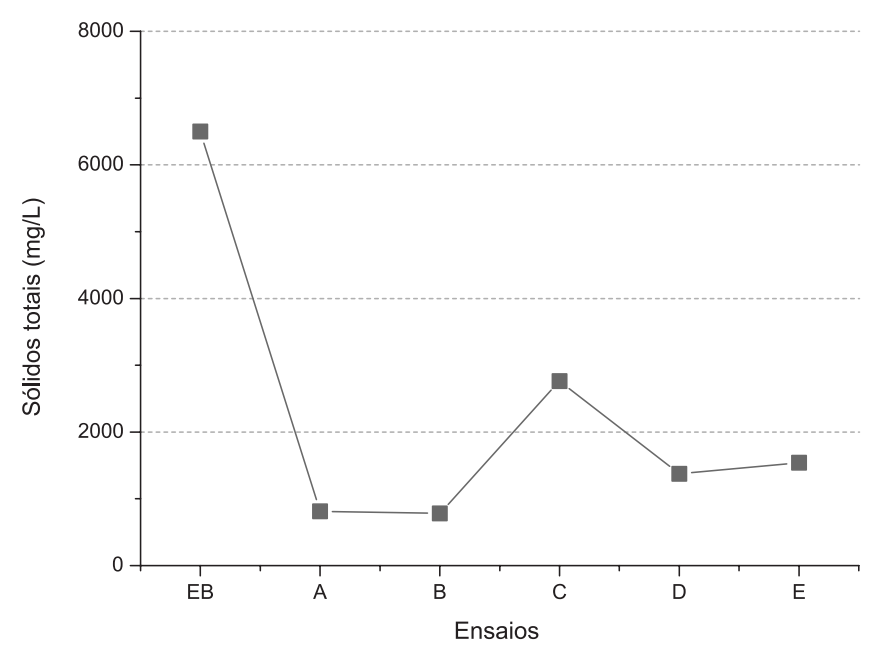

Figura 5. Sólidos totais em função do tipo de efluente

Os ensaios A (814 mg/L) e B (782 mg/L) apresentaram menor quantidade de sólidos totais quando comparados aos ensaios C (2761 mg/L), D (1375 mg/L) e E (1542 mg/L). Vários fatores podem estar relacionados a esse resultado, um destes é a quantidade de metal 
perdida do eletrodo que pode ter ficado no efluente, pois nos ensaios A e B se obteve pequena perda de metal; já o ensaio C apresentou uma maior perda de metal dos eletrodos. Avaliando os resultados em relação ao valor do $\mathrm{pH}$ do efluente, verifica-se a influência deste parâmetro no processo de eletrocoagulação. Pesquisando o diagrama de solubilidade do hidróxido de alumínio verifica-se que a amplitude da hidrólise do $\mathrm{Al}^{3+}$ depende do $\mathrm{pH} .{ }^{38}$ Logo, a hidrólise e polimerização do $\mathrm{Al}^{3+}$ na faixa de $\mathrm{pH}$ de 5-9 formam espécies mononucleares e/ ou complexos alumínio polinucleares como, por exemplo, $\mathrm{Al}(\mathrm{OH})_{3}$, $\mathrm{Al}(\mathrm{OH})^{2+}, \mathrm{Al}_{2}(\mathrm{OH})^{4+}$, e $\mathrm{Al}_{17}(\mathrm{OH})_{32}{ }^{7+}$, os quais são efetivos coagulantes de poluentes. Mas em altos valores de $\mathrm{pH}$ da solução a espécie dominante é $\mathrm{A}(\mathrm{OH})_{4}^{-}$, a qual obviamente não coagula os poluentes e permite uma menor eficiência na floculação. ${ }^{39}$

Apesar de ter ocorrido uma remoção de sólidos totais do efluente bruto, o valor de sólidos totais ainda é elevado nos efluentes tratados e pode ser devido à presença de sais dissolvidos, que dificilmente são removidos. Este resultado também foi verificado por Crespilho et al. ${ }^{15}$ que utilizaram a eletrocoagulação para tratar o efluente da indústria de processamento de coco. Os autores verificaram que a quantidade de sólidos totais ficou em torno de $3000 \mathrm{mg} / \mathrm{L}$ para os efluentes tratados.

\section{Remoção de óleos e graxas}

Na Figura 6 pode ser observado o teor de óleos e graxas em função dos ensaios realizados. O teor de óleos e graxas (TOG) é um parâmetro importante a ser analisado, uma vez que quando se encontra em quantidades elevadas pode ocasionar problemas nas estações de tratamentos de efluentes. Altos teores de óleos e graxas dificultam o tratamento biológico, devido à formação de um filme de óleo na camada superficial que impossibilita a aeração, ${ }^{40}$ ou seja, a transferência do oxigênio da atmosfera para a água é impedida, causando também alteração no ecossistema aquático. ${ }^{12}$

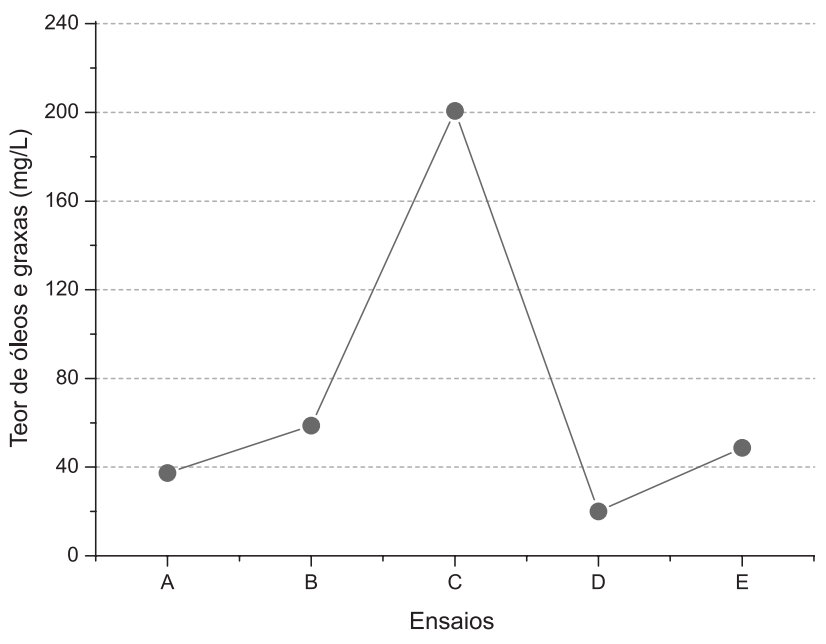

Figura 6. Valores do teor de óleos e graxas em função do tipo de efluente

O efluente bruto utilizado apresentou um alto teor de óleo e graxas, e essa concentração foi reduzida para $37,33 \mathrm{mg} / \mathrm{L}$ (ensaio A), $58,67 \mathrm{mg} / \mathrm{L}$ (ensaio B), $200 \mathrm{mg} / \mathrm{L}$ (ensaio C), 20,00 mg/L (ensaio D) e $48,67 \mathrm{mg} / \mathrm{L}$ (ensaio E). Os ensaios A e D mostraram-se mais eficientes, visto que a Resolução CONAMA estabelece que a concentração de óleos e graxas para lançamento de efluente deve ser inferior a $50 \mathrm{mg} / \mathrm{L}$ para óleos vegetais e gordura animal. ${ }^{9}$ A eletrocoagulação/ flotação removeu em média 99,23\% dos óleos e graxas presentes no efluente bruto, mostrando-se uma técnica bastante eficiente para remoção de óleos e graxas.
Chavalparit e Ongwandee ${ }^{41}$ trataram o efluente do biodiesel com a eletrocoagulação e verificaram que um aumento no tempo de reação conduziu a um aumento na remoção de poluentes e, com a utilização de um $\mathrm{pH}$ no valor de 6 , obtiveram uma remoção de óleos e graxas de $97,83 \%$. Siles et al. ${ }^{42}$ trataram o efluente do biodiesel em um processo combinado de eletrocoagulação e posterior tratamento anaeróbio. Utilizaram a eletrocoagulação para reduzir o teor de óleo do efluente, visto que grandes quantidades de óleo inibem o processo de digestão anaeróbico, e obtiveram uma remoção de $45 \%$ de DQO com a etapa de eletrocoagulação, o que favoreceu o tratamento biológico posterior. ${ }^{42}$

Uma das maiores vantagens da eletroflotação é a remoção de óleos e graxas. ${ }^{14}$ Esse fato ocorre devido à facilidade de coagulação e flotação das moléculas de óleos e graxas. ${ }^{18}$

\section{Consumo de energia}

Está relacionado ao espaçamento entre os eletrodos e o tempo de tratamento. No ensaio A $\left(1,60 \mathrm{kWh} / \mathrm{m}^{3}\right)$ o consumo de energia está relacionado ao tempo de tratamento (40 min) bem como à distância entre os eletrodos ( $8 \mathrm{~mm}$ ), porque para este espaçamento dos eletrodos foi necessária uma voltagem maior $(4,8 \mathrm{~V})$ e, devido a esta voltagem, uma baixa corrente foi requerida no processo e, com isso, o consumo de energia foi baixo quando comparado ao ensaio $\mathrm{C}\left(2,33 \mathrm{kWh} / \mathrm{m}^{3}\right)$ que possuiu um espaçamento menor entre os eletrodos ( $6 \mathrm{~mm})$ e uma menor voltagem foi aplicada $(3,5 \mathrm{~V})$, porém, o aumento do consumo de energia neste ensaio está relacionado com o aumento da corrente aplicada que foi maior do que todos os outros ensaios. Já no ensaio $\mathrm{D}\left(1,40 \mathrm{kWh} / \mathrm{m}^{3}\right)$ a energia consumida foi menor do que nos outros dois ensaios mencionados, devido ao espaçamento dos eletrodos que foi de $4 \mathrm{~mm}$ e, com isto, uma voltagem de $3,5 \mathrm{~V}$ foi aplicada e uma menor corrente foi requerida. No ensaio B $\left(0,65 \mathrm{kWh} / \mathrm{m}^{3}\right)$ o tempo de tratamento foi pequeno (20 min), o espaçamento entre os eletrodos também (4 mm) e isto acarretou um menor consumo de energia. Por fim, no ensaio E $\left(2,16 \mathrm{kWh} / \mathrm{m}^{3}\right)$ o consumo foi elevado, devido ao maior tempo de tratamento utilizado, $60 \mathrm{~min}$.

Diante do exposto verifica-se que o tempo de tratamento, bem como o espaçamento entre os eletrodos afetam no consumo de energia, e estes parâmetros são importantes porque afetam a aplicação econômica do processo de eletrocoagulação/ flotação no tratamento do efluente do biodiesel.

\section{CONCLUSÕES}

A partir dos resultados, pode-se verificar que os parâmetros tempo de tratamento, $\mathrm{pH}$ do efluente e espaçamento dos eletrodos são extremamente importantes no processo de eletrocoagulação/ flotação. Conforme verificado por vários autores que utilizaram esta técnica, ${ }^{14,15,19} \mathrm{o} \mathrm{pH}$ do efluente após o tratamento está relacionado ao $\mathrm{pH}$ inicial e ao tempo de tratamento. Este fator também influencia na remoção de poluentes, visto que a formação do hidróxido $\mathrm{Al}(\mathrm{OH})_{3}$, maior responsável pela remoção de poluentes, ocorre numa faixa de $\mathrm{pH}$ de 5-9. O uso de $\mathrm{pH}$ alcalino induz o ataque químico no catodo de alumínio, devido à evolução do hidrogênio, e isto contribuiu para a quantidade total de alumínio liberado dos anodos. O consumo de energia também está ligado ao tempo de tratamento e ao espaçamento dos eletrodos. Verificou-se que um menor espaçamento é mais indicado para se ter um menor consumo energético. O processo de eletrocoagulação foi capaz de reduzir $99 \%$ da quantidade inicial do teor de óleos e graxas.

Do ponto de vista ambiental, a eletrocoagulação/flotação mostrase como uma técnica bastante promissora, pois o aspecto visual inicial do efluente também foi melhorado, apresentando um efluente tratado 
sem cor aparente. Representa uma grande alternativa ao tratamento do efluente oriundo da etapa de lavagem do biodiesel, sendo uma opção vantajosa do ponto de vista econômico e ambiental.

Diante dos resultados e dos dados obtidos pela pesquisa bibliográfica, um planejamento fatorial pode ser aplicado para avaliar estatisticamente a influência do tempo de tratamento e do valor do $\mathrm{pH}$ inicial do efluente, mantendo-se o espaçamento entre os eletrodos fixo em $4 \mathrm{~mm}$ e voltagem fixa no valor de $3,5 \mathrm{~V}$.

\section{REFERÊNCIAS}

1. Silva, P. R. F. da; Freitas, T. F. S.; Ciência Rural 2008, 38, 843.

2. Gerpen, J. V.; Fuel Process. Technol. 2005, 86, 1097.

3. http://www.anp.gov.br/?pg=46827\&m=\&t1=\&t2=\&t3=\&t4=\&ar=\&ps $=\&$ cachebust $=1306154748318$, acessada em Maio 2011 .

4. Suehara, K.; Kawamoto, Y.; Fujii, E.; Kohda, J.; Nakano, Y.; Yano, T.; J. Biosci. Bioeng. 2005, 100, 437.

5. Berrios, M.; Martín, M. A.; Chica, A. F.; Martín, A.; Chem. Eng. J. 2010, $160,473$.

6. http://www.anp.gov.br/?pg=38591\&m=anuario\&t1 $=\& \mathrm{t} 2=$ anuario $\& \mathrm{t} 3=\&$ $\mathrm{t} 4=\& \mathrm{ar}=0 \& \mathrm{ps}=1 \&$ cachebust=1306155277867, acessada em Maio 2011.

7. Knothe, G.; van Gerpen, J.; Krahl, J.; Ramos, L. P.; Manual de Biodiesel, 1ª ed., Editora Edgard Blücher: São Paulo, 2006.

8. Jaruwat, P.; Sangkorn, K.; Hunsom, M.; Energy Conversion Manag. $\mathbf{2 0 1 0}, 51,531$.

9. www.mma.gov.br/port/conama/res/res05/res35705.pdf, acessada em Junho 2010.

10. Kolesárová, N.; Hutnan, M.; Spaçková, V.; Lazor, M.; $37^{\text {th }}$ International Conference of SSCHE, Tatranski Matliare, Slovakia, 2010.

11. De Boni, L. A. B.; Goldani, E.; Milcharek, C. D.; Santos, F. A.; Tchê Química 2007, 4, 41.

12. Granjeiro, R. V. T.; Dissertação de Mestrado, Universidade Federal da Paraíba, Brasil, 2009.

13. Sinoti, A. L. L.; Souza, M. A. A.; Anais do $23^{\circ}$ Congresso Brasileiro de Engenharia Sanitária e Ambiental, Campo Grande, Brasil, 2007.

14. Chen, X.; Chen, C.; Yue, P. I.; Sep Purif. Technol. 2000, 19, 65.

15. Crespilho, F. N.; Santana, C. G.; Rezende, M. O. O.; Quim. Nova 2004, 27, 387.

16. Mansour, L. Ben; Chalbi, S.; J. Appl. Electrochem. 2006, 36, 577.

17. Daneshvar, N.; Oladegaragoze, A.; Djafarzadeh, N.; J. Hazard. Mater. 2006, 129, 116.

18. Rubach, S.; Saur, I. F.; Filt. Sep.1997, 34, 877.

19. Kobya, M.; Hiz, H.; Senturk, E.; Aydiner, C.; Demirbas, E.; Desalination 2006, 190, 201.
20. Mollah, M. Y. A.; Morkovsky, P.; Gomes, J. A. G.; Kesmez, M.; Parga, J.; Cocke, D. L.; J. Hazard. Mater. 2004, 114, 199.

21. Kobya, M.; Bayramoglu, M.; Can, O. T.; Sozbir, M.; Sep. Purif. Technol. 2004, 37, 117.

22. Barros Neto, B.; Scarminio, I. S.; Bruns, R. E.; Planejamento e Otimização de Experimentos, $2^{\mathrm{a}}$ ed., Ed. da UNICAMP: Campinas, 1996.

23. Koren, J. P. K.; Syversen, U.; Filtration \& Separation 1995, 32, 146.

24. Silva, Paulo C. F.; Dissertação de Mestrado, Universidade Federal Fluminense, Brasil, 2005.

25. Mollah, M. Y. A.; Schennach, R.; Parga, J.; Cocke, D. I.; J. Hazard. Mater. 2001, 84, 29.

26. Holt, P. K.; Barton, G. W.; Michell, C. A.; J. Chemos. 2005, 59, 355.

27. Sivakumar, M.; Emamjomeh, M. M.; J. Environ. Manag. 2009, 90, 1663.

28. Hosny, A. Y.; Sep. Technol. 1996, 6, 9.

29. Ferreira, L. H.; Dissertação de Mestrado, Universidade Federal de Campinas, Brasil, 2006.

30. American Public Health Association, American Water Works Association, Water Environment Federation; Standard Methods for the Exami-



31. Oliveira, D. M.; Dissertação de Mestrado, Universidade Federal de Santa Catarina, Brasil, 2003.

32. Crespilho, F. N.; Rezende, M. O. O.; Eletroflotação - Princípios e Aplicações, $1^{\mathrm{a}}$ ed., Editora Rima: São Carlos, 2004.

33. Mouedhen, G.; Feki, M.; Wery, M. de P.; Ayedi, H. F.; J. Harzad. Mater. 2008, 150,124

34. Picard, T.; Cathalifaud-Feuillade, G.; Mazet, M.; Vandensteendam, C.; J. Environ. Monit. 2000, 2, 77.

35. Can, O. T.; Bayramoglu, M.; Kobya, M.; Ind. Eng. Res. 2003, 42, 3391.

36. Vik, E. A.; Calson, D. A.; Eikum, A. S.; Gjessing, E. T.; Water Res. 1984, 18, 1355.

37. Srirangsan, A.; Ongwandee, M.; Chavalparit, O.; The International Journal Published by the Society of Higher Education Institutes on Enviroment 2009, 2, 15.

38. Kobya, M.; Can, O. T.; Bayramoglu, M.; J. Hazard. Mater. 2003, 100, 163.

39. Holt, P. K.; Barton, G. W.; Wark, M.; Mitchell, C. A.; Colloids Surf. A. 2002, 211, 233

40. Melo, H. N. de S.; Melo, J. L. de S.; Andrade Neto, C. O.; Guimarães, A. K. V.; XXVII Congresso Interamericano de Engenharia Sanitária e Ambiental, Cancun, México, 2002.

41. Chavalparit e, O.; Ongwandee, M.; J. Environ. Sci. 2009, 21, 1491.

42. Siles, J. A.; Martín, M. A.; Chica, A. F.; Martín, A.; Bioresour. Technol. 2010, 101, 6315 . 